\title{
Games Applied to Children with Motor Impairment using the Myo Wearable Device
}

\author{
FLÁVIA G. FERNANDES, ALEXANDRE CARDOSO \& RENATO A. LOPES
}

\begin{abstract}
The characteristic of immersion, involvement and motivation have made serious games an important tool to be used in the medical field. However, there are people that, having some sort of physical disability, are not able to, or do not feel motivated to play. This work presents a strategy to support human- computer interactions for children with a disability in their upper limbs through a wearable device, with the objective of improving access to digital games for the target audience. For the development of this work, the wearable device Myo was used to control a puzzle game as a means of providing the interaction between the individual and the game. In this context, the game is controlled with the movement of the user's upper limb that presents the disability, through the Myo device. For the validation of this research, the game developed was made available for individuals presenting a disability in the upper limbs, from an age range between five and fifteen years old, of the Association of Assistance to the Disabled Child (AACD). Soon, it was noticed that the participants of the research soon could play while utilizing the deficient limb with Myo's support and felt more motivated to play. On the long run, it is expected to contribute to the motivation of patients through technological innovation, so that the process of body consciousness is made more interactive.
\end{abstract}

Key words: physical disability, wearables, digital games, upper limbs.

\section{INTRODUCTION}

According to census data from the Brazilian Institute of Statistical Geography (IBGE) of 2015, there are 200.6 million people with some type of disability in Brazil, corresponding to about 6.2\% of the population. Most of these people define the type of disability as a physical limitation. In this way, it is of great relevance to develop improvements for this class of people, thus contributing to their social inclusion (IBGE 2015).

Often, people with physical or mental disabilities are victims of prejudice and discrimination. They usually do not receive the same type of treatment and have the freedom to come and go damaged by poor conditions of public and private access. However, the Universal
Declaration of Human Rights makes it clear that all people should be treated fraternally, regardless of disability. In the specific case of Brazil, the Federal Constitution defines as a goal the search for the welfare of all, without any kind of discrimination. In the same way, the Brazilian Penal Code determines as punishable the criminal acts and of disrespect caused by discriminatory factors (Guide 2016).

People with physical disabilities in the upper limbs have limitations to perform daily activities (Kouroupetroglou 2013). In this way, they may also have difficulties using technological devices, such as computers, tablets, mobile phones in activities that require two hands, such as digital games. Moreover, the long time necessary for the 
promotion of body awareness, acceptance of disability and the low motivation generated by traditional methods are indicated as a reason for treatment abandonment, being one of the main causes of therapeutic failure (Dias 2009).

Another problem encountered by people with physical disabilities is the lack of resources that allow the continuation of home treatment, with little or no face-to-face monitoring of a therapist (Botella 2010). It is also important to remember that people who are born with congenital malformation of a member of the human body also have other health problems, which may be physical (in other limbs), mental, cardiovascular, respiratory, among others.

On the other hand, there are works, such as PhysioPlay (Santos 2012) and MoVER - Movement in Virtual Environment for Rehabilitation (Sousa Junior 2013), that prove the efficiency of the use of games in the motivation of patients to continue the treatment of physiotherapy of the upper limbs. Both are serious games that simulate physiotherapeutic movements through challenges to perform virtual tasks with the use of the human body, demonstrating the possibility of remote treatment for the promotion of body awareness and its low cost.

Thus, the use of the digital game can become an alternative in providing greater motivation in the games through challenges with virtual techniques, working concepts that can aid in the cognition, in the emotional and physical aspects of the patients, in favoring the movements of the affected limb, during leisure, socialization and coexistence with other children. In addition, the promotion of body awareness in a virtual way through games aims to simulate real situations; it is perceived that the use of it improves the functionality of the affected limbs and leads it to resume activities in the areas of occupational performance (Grande 2011).
In addition, adaptation is important to broaden the cognitive potential of People with Special Educational Needs (PNEES), which is one of the great challenges of inclusion work in the school and social context. However, even with few resources, it is possible to offer good alternatives to meet the peculiarities of people with physical disabilities by adapting everyday materials and equipment. Their use allows people with disabilities to express themselves, to ask questions, to solve problems and to become more participatory, thus allowing greater social interaction with other people.

In this context, the objective of this work is to develop and evaluate a game adapted to be used with Myo, a wearable device that allows the control of applications through the recognition of gestures, to provide better accessibility to people who have some kind of disability in the upper limbs. This work is relevant to encourage the target audience to expand their access to digital entertainment, since digital games may be important for the process of promoting the body awareness of children and adolescents with disabilities in the upper limbs. They can also provide other benefits, such as assisting in the acceptance of motor limitation, motivating patients to use the disabled member more frequently, developing and building skills, potential awakening, learning new technologies, improving cognition, emotional and physical aspects, socialization and leisure.

The works cited confirm that the use of digital games helps to stimulate users to perform real-world activities. In addition, it is known that the use of natural interfaces is recent and stimulating, since they are natural human elements, almost imperceptible when the user is immersed in the application. Therefore, the Myo wearable device is the focus of this work. 


\section{RELATED WORKS}

Among the works available in the literature, some of them present development, concepts and theoretical references related to the subject in question. Thus, some papers were selected to be correlated to this one.

The "Pediatric rehabilitation with the reachman's modular handle", presents the results of a preliminary study with a child with cerebral palsy using the ReHaptic Handle, a new robotic device for pediatric rehabilitation of upper limb function. Interactive computer games were implemented to increase participant engagement and engagement, thus promoting motor recovery. Pinching with forefinger and thumb, forearm supination/pronation as well as flexion/extension of the wrist were trained two to three times a week for fifteen minutes each. There was an increase in the accuracy and smoothness of forearm supination and pronation movement with the subject, as well as a reduction in the duration of movement. Thus, an application is presented for children who have had cerebral palsy, in order to assist them in returning the movements of their upper limbs normally. This prototype is important, since cerebral palsy is a non-progressive neurological disease caused by disorders of the developing brain. Physical and occupational therapy, if started at an early age, can help minimize complications, such as joint contractures, and may improve limb movement and limb coordination. Although current forms of therapy for children with cerebral palsy are effective in minimizing symptoms, many children find them boring or repetitive (Tong 2015).

The "Hand Therapist: a rehabilitation approach based on wearable technology and video gaming" is a hand rehabilitation system, mainly for patients suffering from stroke, consisting of: Myo clamp, robotic glove and a game engine Unity 3D. This approach presents a solution that combines performance, low cost and motivation for hand therapy. Thus, the application is a form of hand therapy aimed at patients suffering from stroke, who do not have physical disabilities, but difficulty in handling objects. In this system, the user performs several repetitive exercises to recover the movements of the hand and, in addition to Myo, use a glove with sensors (Lipovský \& Ferreira 2015).

The "Hand Posture and Gesture Recognition using Myo Armband and Spectral Collaborative Representation based Classification" proposes the use of Spectral Domain-based collaborative representation to recognize the postures and gestures of electromyography (EMG) recordings acquired by a newly introduced sensor: Myo armband Thalmic Labs. The recognition accuracy obtained for a set of six gestures and postures is promising, with an accuracy greater than 97\%, which is an efficient result in related literature. The algorithms are developed to create an intuitive man-machine interface for navigating a robotic wheelchair. This prototype was created for people who use wheelchairs. Thus, the user moves the wheelchair by means of his own gestures of the arm in which the Myo is placed. In this case, the deficiency of people occurs in the lower limbs. In these two projects, the interaction medium could be switched to Kinect and joystick, respectively, which would probably achieve the same result. However, in the work presented here, these other technological tools could not be used, since people have physical disabilities in the upper limbs and have difficulty using the tools cited (Boyali 2015).

The work related and presented in this section contributes to the ratification of the relevance of the use of natural interaction systems to promote upper limb body awareness or alternative interactions. The relevance is emphasized in environments related to the medical field that require a process of 
reproduction or repetition of actions and movements that produce a greater motivational scenario than the traditional methodology.

The work PhysioPlay (Santos 2012) and Mover (Sousa Junior 2013), presented in the Introduction section, present controllers adapted for people with physical disabilities in the upper limbs, similar to this work. However, the present work uses a wearable device for adaption controlled by the user's own gestures, which is something more practical and easy to use, as well as being an innovative and attractive technology for people.

Typical console controllers are equipped with various buttons and joysticks often to be operated at the same time, making it a real barrier. Some do-it-yourself solutions exist adapting business controllers for special needs. However, video game control is left to people with disabilities or physical limitation and often, it is still not able to play the game with its residual features. In addition, such solutions are neither tradable, being uncertified modifications of a commercial device.

The work (Sousa Junior 2013), presents an application for children who had cerebral palsy, in order to help them return the movements of their upper limbs normally. This type of prototype is important, since cerebral palsy is a non-progressive neurological disease, caused by disorders of the developing brain. Physical and occupational therapy, if started at an early age, can help minimize complications, such as joint contractures, and may improve limb movement and limb coordination. Although current forms of therapy for children with cerebral palsy are effective in minimizing symptoms, many children find them boring or repetitive.

The studies (Tong 2015) and (Boyali 2015) present researches using the wearable Myo device. In Lipovský \& Ferreira (2015), the application is a form of hand therapy aimed at patients suffering from stroke, who do not have physical disabilities, but difficulty in handling objects. In this system, the user performs several repetitive exercises to recover the movements of the hand and, in addition to Myo, use a glove with sensors. In (Boyali 2015), the prototype was created for people who use wheelchairs. Thus, the user moves the wheelchair by means of his own gestures of the arm in which the Myo is placed. In this case, the deficiency of people occurs in the lower limbs. In these two projects, the interaction medium could be switched to Kinect and joystick, respectively, which would probably achieve the same result. However, in the present study, these other technological tools could not be used, since people have physical disabilities in the upper limbs and have difficulty using the aforementioned tools.

\section{MATERIALS AND METHODS}

Firstly, in the Conception phase, a bibliographical research was carried out on games applied to the health area and in people with disabilities, game classification and their respective theory of flow - a state of operation in which the person is totally immersed in what he is doing. Flow is characterized by a feeling of total involvement and success in the activity process - types of physical disability in the upper limbs, natural interfaces, wearable devices, among other important concepts to solve the following problem: the difficulty that people with physical disabilities in the upper limbs, mainly children and adolescents, has to use traditional technological devices, often requiring the help of third parties.

After the bibliographical survey and the selection of related works, the Association for Assistance to the Deprived Child (AACD)/ Uberlândia Unit was contacted to better 
understand the problem domain. Together with the institution's team by the coordinator, physicians, physiotherapists, occupational therapists - the project for the evaluation of the Research Ethics Committee (CEP) of the Federal University of Uberlândia was written and submitted through the online system called Plataforma Brasil. The project was submitted to the Brazilian Platform entitled "Serious Games to Support the Rehabilitation of Patients with Physical Disabilities using Natural Interfaces" and CAAE: 55704316.3.0000.5152.

The AACD was chosen as the research coparticipant institution, since it is the closest unit of the Federal University of Uberlândia, where the research was developed, which enables the treatment of promotion of the body's awareness of the target audience of this research: children and adolescents with physical disability in the upper limbs. In addition, it is the most comprehensive organization created to receive the target audience of this research, serving patients from various cities in the region. The purpose of the AACD is to provide improved quality of life and acquisition of greater autonomy and independence for persons with disabilities.

In the Elaboration phase, the Enterprise Architect software was used to model the system architecture and the construction of UML (Unified Modeling Language) diagrams, use cases, classes, activities, functional and non-functional requirements analysis of the application.

In the Construction phase, the Unity 3D software and the $C$ \# programming language (Machado 2011) were used to develop the puzzle game with three levels of difficulty. Subsequently, the Software Development Kit (SDK or Software Development Kit) of the Myo wearable device was modified and implemented in game. The Unity 3D game engine was chosen to be used in this work, since it offers several graphic computing resources necessary for the implementation of the project, besides having a compatibility plug-in with the Myo wearable device. The $\mathrm{C}$ \# programming language was also chosen because it is easily compatible with Unity 3D software.

The decision of a puzzle game was made because this type of game can assist both in promoting the patients' body awareness and in the cognitive aspects, since it exercises the logical reasoning of the users, according to information obtained with AACD professionals.

For the control of the games, the Myo device was used, since it is a model freely marketed not only for researchers, but also for ordinary consumers who wish to use it in computer games or to control other applications. Because it is a non-invasive electromyography method, the Myo device does not cause side effects to the research participant, as it is a watch-like accessory or bracelet, which does not cause allergy, pain, skin irritation, calluses, or any other physical damage or injury. It captures the participant's gestures and then transmits them to the computer, which recognizes the movements and executes the activities during the execution of the games. The construction of the game and the choice of the genre used for this research were monitored by the AACD team, which has greater experience with patients, the target audience of the work.

The symbols shown in Fig. 1 are the main gestures performed by the user during the execution of applications controlled by Myo, according to the wearable device standard.

In this perspective, several tests were performed to understand the operation of the bracelet and the plugins made available for download, in order to know the various possibilities of what can be done with Myo. 


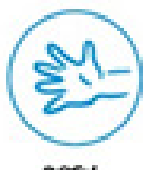

OFEN

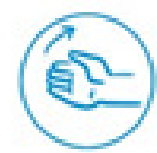

HANC OUT

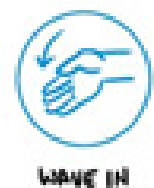

have in

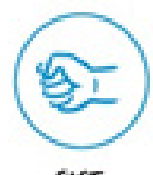

FIST

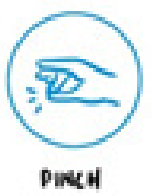

Figure 1. Gestures

executed by the user.
In the puzzle game presented, the "Double Tap" gesture is used to start the game. The "Wave Left" and "Wave Right" gestures are used to select the puzzle pieces left and right, respectively. To move the pieces to the grid, use the "Fist" gesture, and to fit the pieces of the puzzle in the grid, the "Fingers Spread" gesture is used.

After these procedures, it is possible to unlock the software, place Myo on the user's arm, calibrate the device, and use the person's arm movements instead of a mouse and keyboard. Thus, commands that use the mouse and keyboard in the game have been replaced by gestures of the user, so that the system is used with the Myo wearable device.

\section{CONCEPTION AND APPLICATION OF THE TESTS}

First, the AACD surveyed the target audience for the survey: children and adolescents aged 8 to 15 who have physical disabilities in the upper limbs.

In this study, it was found 44 patients who had already attended the institution with this type of disability. Then telephone calls were made to contact the families of these patients and explain about the research. Of the 44 patients, 24 expressed an interest in participating in the study. The other 20 patients did not participate for the following reasons: some were not found, because the telephone number registered in the institution's system no longer exists. Others changed city or state; others live in neighboring cities, but found it difficult to get to the city of Uberlândia for research participation. Some have argued that their children have already been discharged from the AACD, who live well with physical disability in their daily lives and have no interest in participating in the research. Finally, some said the children did not want to participate in the survey.

Thus, an AACD room was prepared to receive the patients and their families to perform the tests with the game controlled by the wearable device Myo. In the dates and schedules marked, ten participants of the survey appeared, where the project was presented in the form of slides to better explain its purpose, and demonstrative video of the operation of the game. Subsequently, the Term of Free and Informed Consent (TCLE) was given to parents and the Term of Assent for children and adolescents, and doubts were resolved about the research project. After reading the TCLEs, they were signed.

Subsequently, the questionnaire on the evaluation of the individual's profile with the researcher's personal data (child or adolescent) was completed. It addresses socio-demographic issues and was elaborated from a questionnaire of internal use of AACD.

The 10 children who participated in the research had congenital physical disabilities (from birth), due to malformation during pregnancy: five children had right hand agenesis (ICD Q71.3 Congenital absence of hand and fingers) and five others had hypoplasia of phalanges (ICD Q71.8 - Other lower limb reduction defects). Children have other deficiencies as well, and may be physical (lower limbs), mental or cardiovascular. 
The tests were performed with an 8 year old child, a 9 year old child, two 10 year old children, two 11 year old children, two 12 year old children and two 13 year old children. Of these children, six individuals were of the female gender and four were of the male gender.

The other questions in the questionnaire assessing the profile of the individuals participating in the research allowed us to identify that the children's preferred genders are adventure games, reasoning and creativity. In addition, it has been found that children are in elementary school, most are in the appropriate school year for their age. However, some have had disappointments and are somewhat behind because they have problems of attention deficit, hyperactivity and learning difficulties.

Children also have limitations to perform daily activities that require both hands, since the 10 children who participated in the research have deficiency in the right upper limb. In this way, they are left-handed and seek to do most of the activities with their left hand.

Also questioned about the degree of experience of children with technology, computers, tablets and mobile phones. The answers indicated that the participants of the research use technological devices in an intensive way, although they have deficiency in the upper limb. However, parents said their children like to use these devices only at home, with their family. In outdoor settings, they are ashamed to show their motor limitation in the upper limb for unknown people due to fear of prejudice and rejection.

In addition, children were asked about the degree of experience of children with digital games and video games. In it, it is observed that there is a moderate use of games. According to parents, children cannot play most digital games alone due to deficiency in the right upper limb, and they are frustrated that they do not have autonomy when performing this practice. The parents then claimed that they do not allow their children to play much to avoid this type of situation, whichcan lead to behavior of low selfesteem of the children and reduction of social interaction.

Then the Myo bracelet was placed on the arm of the research participant so that the controls of the game were controlled by the gestures and movements of the child or adolescent.

Some children had the same behavior when playing with both arms. However, others were more difficult to control the game with the arm that has the disability. This can occur due to several factors, like poor use of the disabled limb and deficient muscular anomalies.

After the game was executed, a questionnaire was applied to the participant of the survey on the evaluation of the use of the game mediated by the Myo wearable device. The service to each research participant was carried out individually. The parents and an occupational therapist working in the AACD followed all procedures.

The questionnaire to evaluate the use of games mediated by the Myo device was elaborated from a questionnaire for evaluation of educational games, proposed by Savi (2010). It was chosen because it addresses a number of important features about gameplay with children, including motivation, attention, relevance, confidence, satisfaction, user experience, immersion, challenge, ability, competence, comfort, interest, fun, learning, and knowledge. In this way, the questionnaire was adapted, reducing it to 20 questions. In addition to the questions already in the questionnaire (Savi 2010), other questions about Myo were included, in order to better address the questions in the tests and to investigate their impact on the research. In addition, this questionnaire was made using the first-person language appropriate for the age group and also 
facial expressions to facilitate the understanding of the children when they respond to the same.

\section{DISCUSSION}

The following are the results obtained from the application of the evaluation questionnaire of the puzzle game through the Myo wearable device:

For the first question of the questionnaire "Did I already knew the device Myo?" all the participants of the research answered "Nothing", which corresponds to $100 \%$ of the sample. Thus, it can be observed that none of the participants of the research knew Myo, that is, the bracelet was a novelty for the children involved in the tests and for their respective parents.

For the $2^{\text {nd }}$ question from the questionnaire "Have I used Myo before?" all the participants answered "Nothing", which corresponds to $100 \%$ of the sample. Thus, it is verified that none of the survey participants ever used the wearable device, that is, this was the first contact of users with Myo.

In Fig. 2, the result for the answers to the third question in the questionnaire is shown. Being "Was it easy to learn how to use Myo to play?", it is observed that the learning to use Myo was moderate, since the presented answers were varied. This was because some children have attention deficit and learning disabilities, correlated with the physical deficiency in the upper limb, which, consequently, can also impair the adaptation to the new method of play.

For Question 4 of the questionnaire, "Was it comfortable to use Myo to play?", all respondents answered "Most", which corresponds to $100 \%$ of the sample. In this way, it can be observed that all the participants of the research appreciated the comfort of the wearable device, since it does not hurt or cause allergies, it is similar to a bracelet or bracelet.

For the $5^{\text {th }}$ question in the questionnaire "Did I like to control the game using Myo?", All the participants answered "A lot", which corresponds to $100 \%$ of the sample. Thus, it is verified that all the participants of the research liked to control the game using Myo, since the possibility of using it to control the commands of the game was a great novelty for the children.

For the $6^{\text {th }}$ questionnaire of the questionnaire "Does Myo met my expectations?", all the participants answered "A lot", which corresponds to $100 \%$ of the sample. In this way, it can be seen that the device met the expectations of all the children who participated in the research. They were very excited when the project was presented to them in the form of slides and video demo. They were also very happy and surprised to learn to play and managed to control the game with their own gestures.

For the $7^{\text {th }}$ question from the questionnaire, "Did I find the game interesting and interesting?", all participants replied "A lot", which corresponds to $100 \%$ of the sample. Thus, it was observed that all participants found the game to be cool and interesting, and they were motivated to execute the game commands using their own gestures, to hit the puzzle pieces in the puzzle and to finish the game.

For the $8^{\text {th }}$ question on the questionnaire, "Was it easy to stay focused on the game?", all respondents replied "A lot", which corresponds to $100 \%$ of the sample. In this way, it is verified that all the participants of the research considered easy to maintain the attention in the game. First, because the children were interested in performing the correct moves with the upper limb to complete the game and achieve higher scores. In addition, the puzzle is a game that requires reasoning and attention to be played. Another important aspect observed was that, 


\section{Wasiteasy tolearn to use Myo to play?}

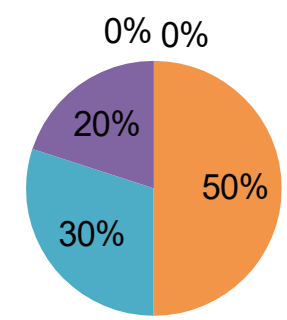

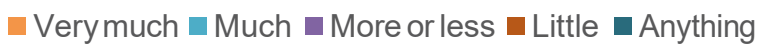

\section{Figure 2. Ease of adaptation to Myo.}

despite the use of the new device for children, since they did not know Myo, and their use by the disabled member, they kept their attention in the game. The interest in playing and completing the levels was an indication that they have been in flow, a state of mind in which the person is immersed in what he is doing, characterized by a feeling of total involvementand success in the process of the activity.

In Fig. 3, the result for the answers to the $9^{\text {th }}$ question in the questionnaire is shown: "Were the game activities easy to understand?". Thus, it is perceived that the participants of the research considered the activities of the game of easy understanding, since the puzzle is a type of game known to them, and the images to be assembled are of animals, something of their knowledge as well.

The Fig. 4 shows the graph for answers to the $10^{\text {th }}$ question in the questionnaire. "Was it easy to learn the commands for running the game?" In this way, it is observed that learning the commands to execute the game was considered moderate, since the children did not know the Myo device nor other technologies that control games by means of gestures. In addition, children can be embarrassed, have motor limitation, and poor frequency of use of the upper limb used to play.

\section{Were the activities in the game easy to understand?}

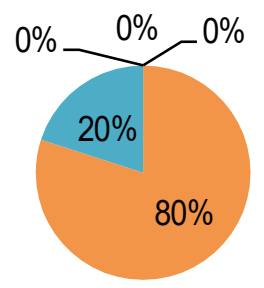

verymuch $\square$ Much $\square$ More or less $\square$ Little $\square$ Anything

Figure 3. Ease to understand the game.

10. Was it easy to learn the commands to run the game?

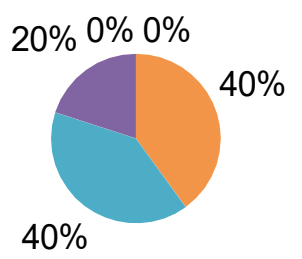

$\square$ Verymuch $\square$ Much $\square$ More or less $\square$ Little $\square$ Anything

Fig. 4. Ease of learning the game's commands.

In Fig. 5, the result for the answers to the $11^{\text {th }}$ question in the questionnaire "Was I able to execute all the game commands?" is displayed. Then, it is noticed that all the participants of the game have been able to execute all the commands of the game, despite the novelty and of initially, do not want to use the member with deficiency to play. However, they later demonstrated skill and satisfaction in playing the puzzle with Myo.

For the $12^{\text {th }}$ question in the questionnaire, "Did I strive to have good results in the game?", all the participants answered "A lot", which corresponds to $100 \%$ of the sample. In this way, it can be observed that the participants of the research struggled to have good results in the 


\section{Was lable to executeall the game commands?}

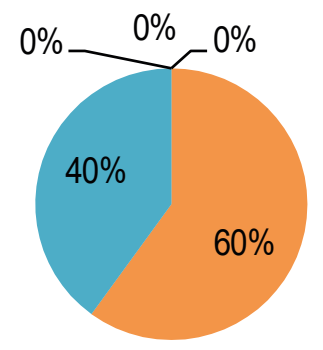

- Very much $\square$ Much $\square$ More or less $\square$ Little $\square$ Anything

\section{Figure 5. Skill with the game.}

game, as they were interested and motivated to play and complete the game with the highest score. The Fig. 6 shows the result for the answers to the $13^{\text {th }}$ question in the questionnaire "Was the speed of execution of the game fast?". Thus, it turns out that the execution speed of the game was medium, since some children took some time to learn the commands of the game through gestures. They were also struggling due to poor limb use and motor impairment.

For the $14^{\text {th }}$ question from the questionnaire "Did Ihave fun during the game?", all participants answered "A lot", which corresponds to 100\% of the sample. In this way, it is noticed that all the participants had fun during the game. Their enjoyment was clear for being able to control the game with their own limb with limitation, they gave a feeling of surprise and they felt useful, which can increase their autonomy and the acceptance of the disability.

For the $15^{\text {th }}$ question from the questionnaire "Was I involved in the game?", all the participants answered "A lot", which corresponds to 100\% of the sample. Then, it is observed that the participants of the research were involved with the game, they felt immersed in the game of puzzle, in flow, that is to say, the correct balance between the challenge inherent to the activity

\section{Was the speed of execution of the game fast?}

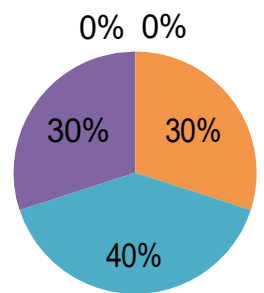

$\square$ Verymuch $\square$ Much $\square$ More or less $\boldsymbol{\square}$ Little $\boldsymbol{\square}$ Anything

\section{Fig. 6. Game pacing.}

and the ability of the player in surpassing it, it. These facts occurred due to familiarity with the images of the game and also to the practicality of the Myo device.

The Fig. 7 shows the graph for answers to the $16^{\text {th }}$ question in the questionnaire "Was I anxious or bored during the game?". In this way, it is seen that most of the participants did not feel anxious or bored during the game, since the Myo device and the possibility of control of the game through it were interesting and interesting novelties for the participants of the research.

For the $17^{\text {th }}$ question from the questionnaire, "Did I feel stimulated to learn from the game?", all the participants answered "A lot", which corresponds to $100 \%$ of the sample. Thus, all the participants of the research felt stimulated to learn from the game, trained their skills and abilities, overcome the challenges and fears, in order to complete the game.

The Fig. 8 shows the graph for the answers to the $18^{\text {th }}$ question in the questionnaire, "Was I able to complete the game?". In this way, one notices that the participants of the research were able to complete the game. The children who selected the option "Much" in this question had difficulties to finish the "Difficult" level of the game, needing the help of the researchers 


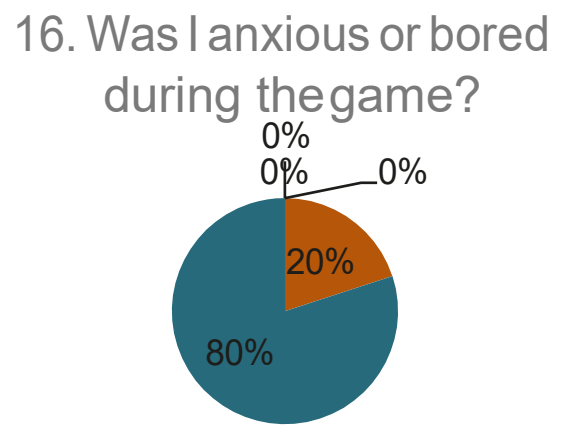

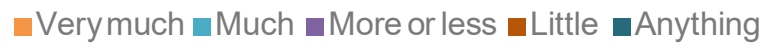

\section{Figure 7. Anxiety or boredom during play.}

to give tips, due to their attention deficit and difficulty concentrating and learning.

In Fig. 9, the result for the answers to the $19^{\text {th }}$ question in the questionnaire "Does the game meet my expectations?". Thus, it is observed that the game met the expectations of the participants of the research. They played with a lot of effort and dedication to learn the commands using Myo and complete the puzzle.

For the $20^{\text {th }}$ question from the questionnaire, "Would I like to play this game again?", all the participants answered "A lot", which corresponds to $100 \%$ of the sample. In this way, it is seen that all the participants in the survey were satisfied and would like to play the puzzle using Myo again. They found a new possibility to play with autonomy, using their disabled member, and without having to rely on other people to help them play. It then demonstrates the importance and relevance of research in the participants' lives.

Among the related works, all approach adaptation of games for upper limbs. The material used for adaptation used for each work was chosen because of adherence to the problem to be solved and informational protection.

The application area of the adaptation, in the great majority of the works, is related to school area, mainly learning and social
18. Was lable to complete the game?

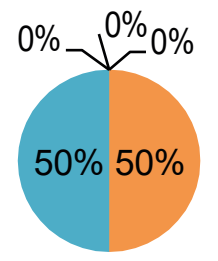

$\square$ Very much $\square$ Much $\square$ More or less $\square$ Little $\square$ Anything

Figure 8. Game clear.

\section{Does the game met my} expectations?

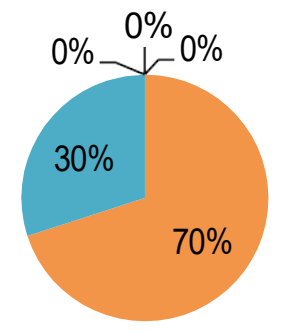

Verymuch $\square$ Much $\square$ More or less $\square$ Little $\square$ Anything

\section{Fig. 9. Game expectations.}

inclusion, demonstrating the importance of the use of alternative procedures to the traditional ones for the most varied types of treatment. And the other jobs involve creating games for rehabilitation of people with physical disabilities or motor impairment due to cerebral palsy or stroke. Another aspect present in this work, which differentiates it from the correlates is the motivational characteristic, in which the game benefits from the digital resources to promote greater immersion and interactivity of the people and, consequently, greater motivation of the users to use the application, which causes results satisfactory in the process.

In this line of reasoning, after the resulting and comparative analysis of the presented works, the contribution of the present work 
appeared: the adaptation of digital games for children who have physical deficiency in the upper limbs, either by congenital and / or acquired malformation, with the purpose of that it is possible to use digital games by this public and also help in the acceptance of the deficiency. This adaptation was made using the wearable device Myo, since it is a bracelet that offers ease and practicality of use. Thus, it is intended to offer these children an additional tool (the digital games) for the process of promoting body awareness and extend their accessibility to games.

\section{CONCLUSIONS}

The results from the tests performed show that games mediated by Myo can be a good alternative to provide accessibility for people with disabilities in the upper limbs. Making digital games more accessible to this audience is relevant as it can provide greater access to digital entertainment, as well as greater development and use of the member, and can aid in the process of promoting body awareness. That way, the benefits, discussed in the literature, that the act of playing can provide people becomes more accessible to people.

The choice of the puzzle game proved adequate for the tests because the participants demonstrated, through their answers, that the game was good, stimulating and met expectations. It was important to choose a simple game because of the age group of the participants, as well as possible neurological problems presented by them. Thus, they could undertake a greater cognitive load in the use of Myo and not in the learning of the game, since the wearable device was a novelty for all participants.

The use of Myo was well accepted. The participants felt comfortable and were able to interact with the game achieving the objectives proposed by the same. Although they presented a moderate difficulty in using Myo, this fact did not interfere in the motivation and involvement of the participants with the game. They reported having had a fun experience while playing. In this way, it can be seen that Myo can be used as a device to improve the accessibility of IASs with disabilities in the upper limbs of digital games, making games more accessible is expected greater use of digital games by the target audience of this research. Thus, they will be able to enjoy the benefits of using the games presented in the literature.

The main difficulties encountered in carrying out this work were the delay in approval of the project by the Research Ethics Committee (about seven months) and the recognition of movements by the wearable Myo device due to its complexity and the diversity of variables involved in this process.

Therefore, it is verified that the objective proposed at the beginning of this work was reached, since the use of games mediated by the wearable Myo device proved efficient in the use of digital games for people with physical disabilities in the upper limbs, providing greater autonomy and accessibility to digital entertainment.

Thus, in the long term, the presented strategy can help in the acceptance of the motor limitation, motivate the patients to use the disabled member more frequently, develop and create abilities, awaken potential, learn new technologies, improve the body awareness process, the emotional, physical and cognitive aspects, socialization and leisure.

Therefore, in view of the results of the tests performed with patients and the questionnaires answered by them, it can be said that it is an innovative therapeutic modality for the promotion of body awareness, and it can also 
encourage the creation of new objects of study and systems in the area doctor.

As future work, it is intended to continue with the selection, development and adaptation of more games with the purpose of improving the incentive to digital entertainment for children and adolescents with physical disabilities in the upper limbs. Also, to monitor the impacts that the use of the game in the long term may entail for the promotion of the corporal consciousness of the individuals, from the point of view physicalmotor and cognitive.

In addition, it is expected to test the puzzle game for use with Myo with more patients with physical disabilities in the upper limbs, to extend the age range of application of the prototype, and to assist the AACD in the inauguration and maintenance of the Virtual Rehabilitation room in the institution in Uberlândia. It is also desired to perform tests with patients with physical disabilities in the two upper limbs to evaluate the results. And later, in these cases, verify the possibility of using two wearable devices (one for each member), and can extend the research to a multimodal analysis strategy.

Still, it is desired to research wearable devices to adapt more games to other members of the human body. If possible, it would be necessary to find generic strategies for using games for all types of disabilities in order to promote the body awareness of patients in this category. Also, to carry out the development of an application supervision module, in which the skilled professional could view graphs to follow the evolution of determining patient, and also compare the results obtained by different patients in the execution of the game.

\section{REFERENCES}

BOTELLA C, BRETÓN-LÓPEZ J, QUERO S, BAÑOS R \& GARCÍAPALACIOS A. 2010. Treating cockroach phobia with augmented reality. Behav Ther 41(3): 401-413.

BOYALI A, HASHIMOTO N \& MATSUMOTO O. 2015. Hand Posture and Gesture Recognition using Myo Armband and Spectral Collaborative Representation based Classification. In: IEEE $4^{\text {th }}$ Global Conference on Consumer Electronics - GCCE.

DIAS RS, SAMPAIO ILA \& TADDEO LS. 2009. Physiotherapy: The Introduction of Playful in the process of rehabilitation of patients in physiotherapeutic treatment. In: VIII Brazilian Symposium on Games and Digital Entertainment. 4. Rio de Janeiro-RJ.

GRANDE AAB, GALVÃO FRO \& GONDIM LCA. 2011. Virtual rehabilitation through videogame: case report in the treatment of a patient with high lesion of the median and ulnar nerves. Rev Acta Fisiat 18(3): 157-162..

GUIDE OF RIGHTS. 2016. Discrimination with the Disabled. Available at:<http://www.guiadedireitos. org/index.php?option=com_content\&view=ar ticle\&id=1040\&Itemid=264>. Accessed on: 20 Dec. 2018.

IBGE - BRAZILIAN INSTITUTE OF GEOGRAPHY AND STATISTICS. 2015. Available at: <http://www.ibge.gov.br/home/ estatistica/populacao/estimativa2015/>. Accessed on: 15 Dec. 2018.

KOUROUPETROGLOU G. 2013. Disability Informatics and Web Accessibility for Motor Limitations. IGI Global.

LIPOVSKÝ R \& FERREIRA HA. 2015. Hand Therapist: a rehabilitation approach based on wearable technology and video gaming. Portuguese BioEngineering Meeting. 4. Port: Portugal.

MACHADO LS, MARQUES FLSN, COSTA RMEM \& MORAES RM. 2011. Virtual reality for health in Brazil: concepts, challenges and opportunities. Braz J Biomed Eng 27(4): 243-258.

SANTOS JVS, CARVALHO LC \& BRESSAN PA. 2012. Physioplay: an exergame for physical rehabilitation applying the interactivity of Kinect as visual biofeedback. In: IX Virtual and Augmented Reality Workshop - WRVA, Paranavaí.

SAVI R, WANGENHEIM CG \& BORGATTO AF. 2010. Proposal of an evaluation model of educational games. New Technologies in Education 8(3).

SOUSA JUNIOR VD, MONTEIRO CBM, NAKAMURA R, YOJO LS, ARAÚJO LV \& NUNES FLS. 2013. Mover: Serious Game applied to motor rehabilitation using Kinect motion sensor. In: Congress of the Brazilian Society of Computing - CSBC, Maceió. 
TONG LZ, ONG HT, TAN JX, LIN J, BURDET E, GE SS \& TEO CL. 2015. Pediatric rehabilitation with the reachMAN's modular handle. Procedures IEEE Eng Med Biol Soc: 3933-3936.

\section{How to cite}

FERNANDES FG, CARDOSO A \& LOPES AC. 2020. Games Applied to Children with Motor Impairment using the Myo Wearable Device. An Acad Bras Cienc 92: e20190273. DOI 10.1590/0001-3765202020190273.

Manuscript received on March 18, 2019;

accepted for publication on May 7, 2019

\section{Flávia Gonçalves Fernandes ${ }^{1}$}

https://orcid.org/0000-0001-5077-2226

\section{Alexandre Cardoso ${ }^{2}$}

https://orcid.org/0000-0002-2023-9647

\section{Renato de Aquino Lopes ${ }^{3}$}

https://orcid.org/0000-0002-9180-6637

${ }^{1}$ Department of Computing, Federal University of Goiás (UFG), Av. Dr. Lamartine Pinto de Avelar, 1120, Bloco M, Setor Universitário 75704-020 Catalão, GO, Brazil

${ }^{2}$ Faculdade de Engenharia Elétrica - Universidade Federal de Uberlândia (UFU), Av. João Naves de Ávila, 2121, Santa Mônica, 38400-902 Uberlândia, MG, Brazil

${ }^{3}$ Faculdade de Computação, Universidade Federal de Uberlândia/UFU, Av. João Naves de Ávila, 2121, Santa Mônica, 38400-902 Uberlândia, MG, Brazil

Correspondence to: Flávia Gonçalves Fernandes

E-mail: flavia.fernandes92@gmail.com

\section{Author contributions}

The author Flávia Gonçalves Fernandes is responsible for the development and execution of the research, as well as for the writing of the article. Authors Alexandre Cardoso and Renato de Aquino Lopes are advisor of the research project and are responsible for reviewing the article.

\section{(cc) BY}

